\title{
COVID-19 lockdown and lifestyles - A narrative review
}

Running title: COVID-19 lockdown and lifestyle changes

Authors:

Sathyanarayanan Doraiswamy MBBS, MD, DHealth ${ }^{1}$, Sohaila Cheema, MBBS, MPH, CPH ${ }^{1}$, Ahmad Al Mulla MD, MPH, DrPH ${ }^{2}$, Ravinder Mamtani MD, MSc, FACPM, FACOEM, ABoIM $^{1}$

sdo4003@qatar-med.cornell.edu;

soc2005@qatar-med.cornell.edu;

almulla@hamad.qa;

ram2026@,qatar-med.cornell.edu

\section{Affiliation:}

${ }^{1}$ Institute for Population Health, Weill Cornell Medicine - Qatar

${ }^{2}$ Department of Medicine, Hamad Medical Corporation, Qatar

\section{Corresponding author:}

Dr. Sathyanarayanan Doraiswamy

Institute for Population Health

Weill Cornell Medicine - Qatar

Education City, Qatar Foundation

Doha, Qatar

P.O. Box 24144

Telephone: +97444928316

Fax: +974 44928333

Email: sdo4003@qatar-med.cornell.edu 


\begin{abstract}
Background: While controlling the COVID-19 pandemic has been the primary objective worldwide, its effect on human behavior, altered lifestyles, and in turn, the impact on chronic non-communicable diseases cannot be ignored. Ten months into the pandemic, muchpublished literature has emerged on altered lifestyles following the lockdown measures imposed by governments all over the world to control the virus spread.
\end{abstract}

Objective: In this narrative review, we explore the impact of lockdown measures on the six lifestyle factors - namely, diet, physical activity, sleep, stress, social connectedness, and the use of tobacco, alcohol, or other harmful substances.

Methods: We searched PubMed and the World Health Organization's global literature database on COVID-19 and included 298 articles. Using a meta-ethnographic approach, we provide an interpretative synthesis of relevant articles.

Results: Among the six lifestyle factors, most of the retrieved articles focused on stress, emotional well-being, and physical activity. Fewer articles discussed alterations in dietary habits, sleep patterns, and the use of tobacco, alcohol, and other harmful substances. Most of the articles included in our review identified the negative effect of lockdown measures on each of the lifestyle factors in many parts of the world. Interestingly, encouraging lifestyle trends were also highlighted in a small number of articles included in our review. Such trends can positively influence the outcome of lifestyle-related chronic diseases, such as obesity and diabetes.

Conclusions: These unprecedented circumstances provide an opportunity to better understand the negative impact of strict lockdown measures on lifestyles, and at the same time, help identify and initiate positive behavioral changes, which if consolidated, may improve chronic disease outcomes in the long run. It would be up to governments, communities, and academia to learn - and benefit from lessons learned from the COVID-19 pandemic - with the ultimate objective of better educating and promoting healthy lifestyles among communities.

Keywords: COVID-19, Lifestyle, Diet, Physical activity, Stress, Smoking, Substance, Alcohol, Emotional well-being, Social connectedness 


\section{Background}

The ongoing COVID-19 pandemic has caused thousands of deaths, overloaded health systems, and disrupted social and economic infrastructure worldwide. While the world remains focused on controlling the pandemic, its impact on lifestyles and in turn, on chronic non-communicable diseases (NCDs) which account for over $70 \%$ of the global mortality, cannot be ignored. COVID-19 controls, requiring people to stay at home and practice physical distancing to prevent transmission, call for greater attention to lifestyle factors and understanding their effect on NCDs.

Various degrees of lockdown measures, including restricting travel and movement, have helped reduce the virus spread. However, these measures also diminish the access and attraction of people to healthy food; widen inequities between communities; result in suboptimal physical activity levels; exacerbate anxiety and stress; impair the quality of optimum sleep; and, encourage substance abuse to allay anxiety and fear of the disease ${ }^{1}$. A large body of published literature has emerged on altered lifestyles since the onset of the pandemic. To our knowledge, there is no synthesis of this emerging literature on altered lifestyle factors during the pandemic.

Given the wide scope of lifestyle-related factors, we conducted a narrative review to help understand these further in the realm of COVID-19. We aim to synthesize the alteration (positive or negative) in lifestyle factors among individuals and communities owing to the effects of lockdown measures implemented by various countries. This narrative review can be an instructive and valuable source for stakeholders interested in weighing the effect of lockdown measures on lifestyles. Additionally, it can inspire further research in the emerging field of lifestyle medicine and its potential effect before, during, and after pandemics such as COVID-19. For the purpose of our review, we recognize the American College of Lifestyle Medicine (ACLM) definition of a healthy lifestyle that includes eating a predominantly plantbased diet, being physically active, sleeping well, managing stress, remaining socially connected, and refraining from the use of tobacco, alcohol, and other harmful substances. When optimally practiced, these six lifestyle factors can prevent, treat, and in some cases even reverse chronic disease. Our narrative review will focus on these six lifestyle factors and how they may have been altered during the COVID-19 pandemic.

\section{Methods}

We conducted the narrative review utilizing a best-evidence synthesis approach put forward by Green et.al ${ }^{2}$. A successful narrative review following this approach is expected do the following: "present information that is written using the required elements for a narrative review, be well structured, synthesize the available evidence pertaining to the topic, and convey a clear message" 2 . Following the Scale for the Assessment of Narrative Review Articles (SANRA) ${ }^{3}$, we have reported our methodology with the checklist included as Appendix 1.

\section{Search strategy}

The search strategy included both keywords, as well as controlled vocabulary (such as MeSH terms). The keywords included the following: COVID-19 AND (lifestyle OR dietary habits OR physical activity OR sleep OR tobacco OR alcohol OR substance OR stress OR relationships) AND (lockdown OR quarantine OR isolation). We searched using these terms in PubMed and the World Health Organization (WHO) global literature database on coronavirus disease with no restrictions on language, geography, or publication date. The reference lists of the retrieved articles were scanned for additional articles relevant to our review. 


\section{Inclusion and exclusion criteria}

We included all articles that discussed lifestyle in the context of the COVID-19 pandemic. The review focused on the six lifestyle factors included in the definition of a healthy lifestyle by the American College of Lifestyle Medicine.

Articles were excluded if they did not focus specifically on any of the six lifestyle factors and did not discuss about lifestyle within the COVID-19 context. Additionally, we excluded published literature describing modelled effects of lockdown during COVID-19.

\section{Information extraction}

Identified articles meeting our inclusion criteria were imported into Endnote X9 $9^{4}$. One reviewer screened the title and abstract of the identified articles. From the included articles, the authors extracted qualitative and quantitative information on the COVID-19 related lockdown and its effects on six lifestyle factors (diet, physical activity, stress, connectedness, sleep, and tobacco or other substance use). For primary studies with quantitative data, we paid specific attention to the study design, results, and the conclusion. For articles other than primary studies, we focused on the discussion and conclusion sections. For the qualitative studies, opinions/perspectives, and viewpoints, we have taken a meta-ethnographic approach to provide an interpretative synthesis. Though the ACLM definition combines smoking, alcohol, and substance use as one lifestyle factor, we looked at them separately to explore them in greater depth. When articles studied more than one lifestyle factor and discussed the impact of lockdown measures on patients with pre-existing NCDs, we grouped them under articles that discussed multiple factors. The information extracted from the included articles was classified according to the six lifestyle factors and summarized below:

\section{Results}

Our original search yielded a total of 1286 articles. After title, abstract and full-text screening, 298 articles were found to be relevant to the scope of our narrative review. Figure 1 shows the number of relevant articles discussing various lifestyle factors. Among the six lifestyle factors, the retrieved articles predominantly focused on stress, emotional well-being, and physical activity. A smaller number of studies were identified for change in dietary habits, sleep patterns, and the use of tobacco, alcohol, and other harmful substances. Articles with quantitative data are referred to as 'studies' in the manuscript. Such data was available in $160 / 298(54 \%)$ of the included articles 
Figure 1. Number of articles grouped according to lifestyle factors

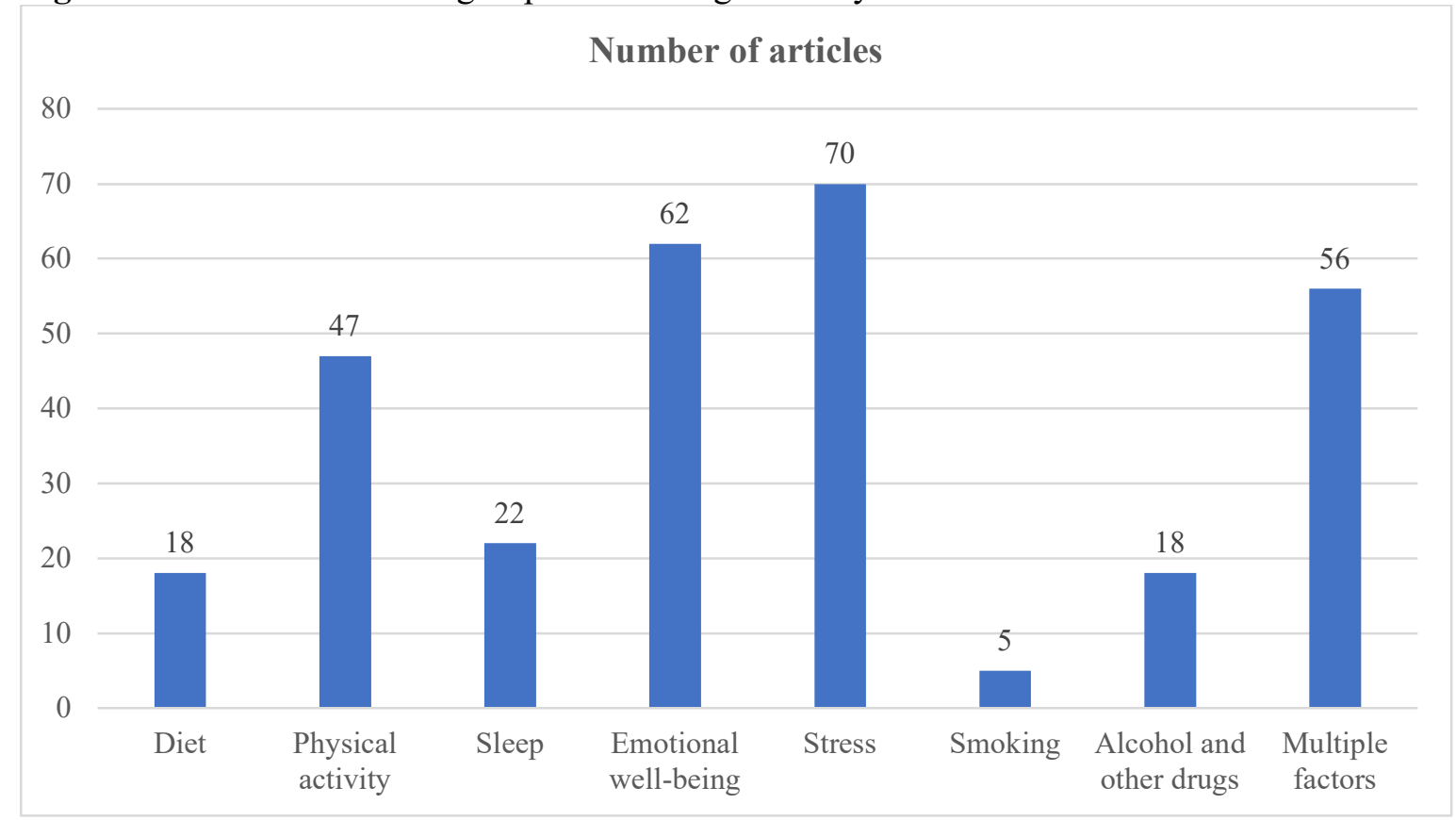

\section{Dietary habits}

Most of the literature included in our narrative review with reference to dietary habits indicates an alteration in the eating behaviors of people in many parts of the world. Most survey-based studies report an increase in the intake of unhealthy foods by the majority of respondents, while a small number demonstrate a higher intake of healthy foods. A Polish study including 2,381 participants found that $34 \%$ of respondents reported eating more during the lockdown ${ }^{5}$, while another study from the same country involving 1,097 participants found $30 \%$ of the participants reported weight gain during the lockdown ${ }^{6}$. Whereas, among 3,533 respondents from Italy, $46.1 \%$ reported consuming more food and $19.5 \%$ had gained weight ${ }^{7}$. Staying home has limited access to fresh vegetables and fruits, particularly in the most vulnerable and poorest sections of the society due to limited availability, accessibility, and affordability ${ }^{5,8}$. In richer households, staying home encouraged excessive use of unhealthy foods, such as processed and fast foods in many parts of the world ${ }^{6,9,10}$. An Italian study reported an increased uptake of 'comfort foods' including chocolates and ice-creams used by participants to allay anxiety caused by the lockdown ${ }^{7}$. Additionally, to overcome boredom, some people turned towards snacking which led to excessive weight gain relatively quickly $6,7,11$.

Interestingly, in some situations staying home encouraged many individuals to eat fresh home-made food, as evidenced by the reduction in the purchase of ready-made meals and by the dominance of in-person grocery shopping as the major source of food and food purchases 7,12. A large study of 7,514 participants from Spain found residents reporting a higher intake of fruits, vegetables, or legumes. The study participants were predominantly young university educated females ${ }^{13}$. Gender (females faring better), family members staying at home, (less) TV watching while eating, geography, and (higher) maternal education have been determined to be factors influencing healthy food intake ${ }^{14}$. In our review, we found that individuals who were overweight/obese pre-pandemic gained more weight, while the undernourished lost more weight ${ }^{6}$. Studies also report that individuals with eating disorders diagnosed prior to the 
lockdown fared worse during the lockdown period due to low self-directedness and less adaptive coping ${ }^{9,15}$.

\section{Physical activity}

The lockdown period saw a general reduction in physical activity levels as evidenced from the studies included in our review. A study from China of 339 participants revealed a significant reduction in the average exercise intensity for both males and females during the lockdown period (average steps per day: before vs during $7038 \pm 1923$ vs $3741 \pm 1042$ step, $\mathrm{P}<0.001$ and $8321 \pm 3000$ vs $3728 \pm 1726$ step, $\mathrm{P}<0.001$ for female and male, respectively) ${ }^{16}$. A similar observation was also made in Italy where $68 \%$ of participants in a study with a sample size of 490 adults reported a decrease in their exercise levels ${ }^{17} .43 \%$ of respondents in a Polish study sample of 2381 also reported a reduction in their physical activity during the lockdown period. An international, multi-country online survey of 1,047 participants found that the number of days per week of all physical activity declined by $24 \%$ during the lockdown period. The number of minutes per day spent on physical activity decreased by $33.5 \%$ while the sitting time had increased 5-8 hours among the respondents ${ }^{9}$. The working from home environment has predisposed individuals to spend more time sitting, leaning, reclining or lying down on the bed ${ }^{9}$. There is no predictable pattern as to who were less active during the lockdown period. While some studies found previously physically active individuals became less active during the lockdown period ${ }^{18,19}$, other studies found no change ${ }^{18}$, or further reduction in physical activity among previously inactive individuals ${ }^{20}$. A cross-sectional study across 64 cities in China with 369 participants, concluded that previously physically active individuals were in greater distress as a consequence of the lockdown ${ }^{21}$. It was also observed that older people, those who were used to exercising in groups, and others who were not accustomed to online apps (which support exercise routine), suffered more with the reduction in physical activity levels ${ }^{19}$.

Lockdown has also provided an opportunity to be creative in finding ways to stay physically active. In South Korea, for example, it was observed that the public bicycle sharing system recorded an increase in the commuters and weekend users ${ }^{22}$. It is, however, to be noted that such initiatives require commitment from the citizens and authorities in ensuring adherence to physical distancing, hand hygiene, use of facemasks, and cleaning of public facility/site surfaces. Staying physically active in home environments is possible and its feasibility has also been shown during the lockdown period in countries like Japan ${ }^{23}$. Studies done during the COVID-19 pandemic have reconfirmed that individuals who remained physically active during the lockdown period had lower odds of suffering from stress, anxiety, and depression ${ }^{24}$.

\section{Sleep}

Stay-at-home restrictions made many people experience insomnia as evidenced from the studies included in our review. In a large cross-sectional study across France, two weeks after the lockdown, $74 \%$ of the 1,005 nationally representative participants reported trouble sleeping when compared to $49 \%$ in the last survey pre-lockdown ${ }^{25}$. A study including 400 university students and administrative staff in Italy found $40 \%$ of the respondents suffering from insomnia while $15 \%$ specifically had trouble in sleep initiation ${ }^{26}$. Factors found to be associated with disturbed sleep were increased blue light exposure from extended screen time scanning for COVID-19 updates, browsing the internet to keep busy, and working remotely from home ${ }^{25}$, 27. Respondents in some studies reported sleeping more during the lockdown period. Even among those who slept more, the reported sleep quality was poor ${ }^{28,29}$. Those who slept more also reported experiencing sleepiness during the day and increased daytime sleep duration in a 
study on 203 corporate professionals and 325 university students in India ${ }^{30}$. In the same study, it was also observed that females, younger people, and those experiencing loneliness and COVID-19 related worries had greater difficulty with sleep ${ }^{30}$. One study reported an increased frequency in the uptake of sleeping pills ${ }^{25}$. Increased stress due to COVID-19, information overload, inconsistent bedtime, and low mood are all contributors to insomnia and its harmful effects on health ${ }^{31}$.

Individuals with previously erratic sleep patterns and social jetlag, on the other hand, were provided with an opportunity to normalize their sleep patterns and improve sleep hygiene for optimum functioning as indicated by a few studies ${ }^{32-34}$. One study from the United States of America found that the reduced rigid workload and school schedules actually led to improvement in the overall sleep quality for some individuals ${ }^{35}$.

\section{Substance use}

\section{Tobacco}

While studies on smoking behavior during the COVID-19 lockdown period were few, we found a mixed pattern in the available studies. A cross-sectional study involving 345 American participants found half of the respondents retaining their pre-lockdown level of smoking, a quarter of them reducing their smoking levels, and the rest actually increasing the amount ${ }^{36}$. The same study also found that while $20 \%$ of respondents showed an increased motivation to quit, $15 \%$ showed a reduction in motivation ${ }^{36}$. In a multi-country study involving 6,800 combustible and e-cigarette users from Italy, India, South Africa, the United Kingdom (UK), and the United States of America (USA), indoor smoking had increased in India and Italy, thereby increasing the risk of secondhand smoke exposure among family members ${ }^{37}$. There was a wide variation in people's beliefs from the countries in this study pertaining to the association of smoking with COVID-19. In India, a substantial proportion of the respondents believed that smoking increased the risk of COVID-19, while in Italy, the majority of those surveyed believed that there was no relation between COVID-19 risk and smoking. Results from other countries were inconclusive. Anxiety, boredom, and stress as experienced by many people during COVID-19 contributed to increased smoking in some individuals ${ }^{6,38}$.

Despite many deterrents, motivated individuals saw the lockdown period as an opportunity to become healthy and quit smoking ${ }^{39}$. The motivation to quit was more discernible among those who smoked tobacco cigarettes than electronic cigarettes ${ }^{36}$. Smokers who had a family member or someone close to them suffer from COVID-19 displayed a greater motivation to quit ${ }^{36}$. Many parents ideally want to appear on their best behavior in front of their children and it is postulated as one of the reasons for them to cut down on their cigarette intake while spending more time at home ${ }^{40}$.

\section{Other harmful substances}

Among other substances that cause dependence, alcohol stands out as one of the most commonly used psychoactive substance during the lockdown period ${ }^{41}$, in spite of alcohol supply not being assured in many countries. A study from India documented that alcohol shop closures led to alcohol-dependent individuals experiencing withdrawal symptoms and suicides 42. Where alcohol was available, people resorted to increased alcohol intake to overcome adverse psychological outcomes of the lockdown including stress and insomnia ${ }^{43}$. Data from the Office for National Statistics in the UK demonstrates that alcohol sales rose by over 30\% in month on month volume. In a survey of 6,416 people from China, $32 \%$ of regular alcohol drinkers reported increasing their consumption during the lockdown period ${ }^{44}$. Interestingly, in a survey of 1,555 people in the UK, one-third reported that they had stopped or reduced 
drinking during the lockdown while one-fifth drank more frequently during the same period ${ }^{45}$. A large study from Australia found that people who prior to the pandemic were heavy drinkers, middle-aged, and had higher incomes, increased their drinking during the pandemic ${ }^{46}$. This study also found that individuals who had experienced recent job loss, those who were stressed/depressed and had altered eating and sleep habits consumed larger amounts of alcohol. It was identified that those who consume alcohol found it more difficult to cope with the mental health impact of the pandemic and the associated lockdown ${ }^{41}$.

In addition, the European Monitoring Centre for Drugs and Drug Addiction determined that people using drugs may face additional risks during the crisis, particularly because of the ageing cohort of opioid users and the associated comorbidities ${ }^{47}$. Though the data is sparse at the present time, most experts agree that movement restrictions reduced the availability of illicit drugs ${ }^{48}$, thereby increasing the demand for de-addiction services. Limited availability of these services added to the despair of the addicted population. There was one study from France, however, which found an increase in cannabis use among 31.2\% (195 of 620) of regular cannabis users making up $5.44 \%$ (620 of 11,391$)$ of total participants, increased usage. ${ }^{49}$

\section{Stress}

Watching, reading, or listening to the constant stream of COVID-19 related news led to increased anxiety among individuals and families ${ }^{50}$. A study from China reported the psychological impact of COVID-19 expressed as mild-to-severe anxiety among $25 \%$ of 7,143 college students ${ }^{51} .65 \%$ of 432 respondents in another study from Hong Kong reported clinical levels of depression, anxiety, and/or stress. Older adults, especially those in isolation and with cognitive decline, were identified to be more prone to anxiety, stress, and depression ${ }^{50}$. Articles also suggest that quarantined healthy adults suffer from symptoms of ill mental health like post-traumatic stress, anger, and confusion ${ }^{52}$. We found few studies that were able to make a clear distinction between the stress levels experienced by the general population as part of the community quarantine vs. those who were quarantined as part of contact tracing or after testing as COVID-19 positive. Few studies also seem to suggest greater stress experienced by both those under isolation ${ }^{53}$ and others under home quarantine as part of contact tracing ${ }^{54}$. One study from China, however, found that home self-quarantine was associated with an increased happiness as compared to those who were in community quarantine ${ }^{55}$.

Stress levels have been higher in some groups, such as ,women (particularly pregnant women), older people (particularly with pre-existing mental health conditions), the economically deprived, and those with chronic medical conditions ${ }^{50,56-58}$. Extreme stress driven by the fear of COVID-19 infection, financial crisis, loneliness, pressure to quarantine and isolate when positive, and the unavailability of alcohol have been identified as drivers of suicidal ideation and suicides during the pandemic and the associated lockdown ${ }^{59,60}$. A healthy diet and refraining from reading updates about COVID-19, were very often shown to be the best predictors of lower levels of anxiety and depressive symptoms ${ }^{61}$.

\section{Emotional wellbeing and social connectedness}

Restriction of movement during the pandemic has led to significant reduction in social activity among family, friends/neighbors, and for entertainment ${ }^{62,63}$. This reduction contributed to diminished life satisfaction in over $30 \%$ of participants in a large, multi-continent study involving 1,047 participants ${ }^{63}$. Prolonged social media use has had a deleterious effect on the overall social well-being of individuals as reported by a study in Saudi Arabia ${ }^{62}$. Studies have found that the lack of social interaction has a more profound effect on people with higher income and education ${ }^{62}$. Lack of social interaction also has a negative impact as more time 
passes for older people, as evidenced by a large cross-sectional study in the UK ${ }^{64}$. Children have reported being more restless, irritable, and inattentive during the lockdown with increased screen time being the likely contributor ${ }^{65}$. Girls and women confined to homes and quarantine facilities face increased family tensions, an overload of domestic work, and remain vulnerable to physical and emotional abuse ${ }^{66}$. Intimate partner violence, characterized by physical, sexual, or emotional violence between partners, is also of concern in these challenging times of staying home and physical distancing ${ }^{67}$.

\section{Lifestyle of patients with chronic diseases}

Studies show that patients with pre-existing, non-communicable diseases have found it difficult to maintain a healthy lifestyle ${ }^{68}$. Patients with coronary heart disease, diabetes, and cancer have had to compromise on their physical activity routine and dietary pattern in particular ${ }^{69}$, ${ }^{70}$. In a study from France, $45 \%$ of 195 patients with chronic heart disease had $>25 \%$ reduction in physical activity and $24 \%$ of patients had gained $>2 \mathrm{kgs}$ of weight ${ }^{71}$. Patients fearful of acquiring COVID-19 infection have also been found to postpone medical care for their chronic problems, leading to greater physical and psychological distress while adding to the suffering of several chronically ill patients $56,68,72$.

\section{Discussion}

Our narrative review highlights altered lifestyles of people worldwide during the lockdown period of the pandemic. Both positive and negative changes in the six lifestyle factors (diet, physical activity, stress, connectedness, sleep, and tobacco or other substance use) have occurred, and their impact has varied in different instances and they are summarized in Table 1. These findings could be instructive to health practitioners and health departments in their strategies to prevent chronic diseases.

For dietary habits, at the start of the pandemic, the United Nations Systems Standing Committee on Nutrition warned that the COVID-19 pandemic would disrupt food systems globally. The committee predicted a deterioration in the nutritional status of individuals due to altered dietary practices ${ }^{73}$. Particular concern was expressed over 'food environments', with both external (availability and quality) and internal dimensions (accessibility, affordability, acceptability, and desirability) of the food systems ${ }^{73}$. Recent observations, nine months after the onset of the pandemic, now appear to confirm earlier fears of malnutrition and disrupted food environments, particularly in disadvantaged communities ${ }^{74}$. In general, the availability of healthy, plant-based food is ample in well-to-do communities. However, access to such food during the pandemic could be limited, at least in some settings and for specific populations. 
Table 1. COVID-19 related lockdown and lifestyle influence

\begin{tabular}{|c|c|c|}
\hline Lifestyle factor & Positive factors & Negative factors \\
\hline Healthy diet & Home-food & $\begin{array}{l}\text { Non-availability of vegetables } \\
\text { and fruits; reliance on } \\
\text { processed foods }\end{array}$ \\
\hline Physical activity & $\begin{array}{l}\text { Exploring new opportunities for } \\
\text { remaining physically active in a } \\
\text { home-environment }\end{array}$ & $\begin{array}{l}\text { Closure of gymnasiums and } \\
\text { parks; limited time for } \\
\text { physical activity }\end{array}$ \\
\hline Sleep & $\begin{array}{l}\text { Opportunity to regularize sleep } \\
\text { cycle }\end{array}$ & Excess screen-time and stress \\
\hline $\begin{array}{l}\text { Tobacco, alcohol and } \\
\text { substance use }\end{array}$ & $\begin{array}{l}\text { Reduced availability and } \\
\text { motivation to quit }\end{array}$ & $\begin{array}{l}\text { Stress, anxiety and boredom } \\
\text { increasing use }\end{array}$ \\
\hline Emotional well-being & Increased family time & $\begin{array}{l}\text { Domestic violence; family } \\
\text { tensions; and women abuse }\end{array}$ \\
\hline Social connectedness & Opportunity for acts of kindness & Loneliness; social isolation \\
\hline
\end{tabular}

Lack of access may present health risks--such as lower immunity and adverse chronic disease outcomes--to individuals in those settings.

Alternatively, staying at home has encouraged individuals to eat fresh, homemade food among some populations ${ }^{75}$, likely due to additional time available to cook. Home-cooking not only helps cut down the intake of unhealthy processed and fast foods, but also has proven emotional and health benefits ${ }^{76}$. Further research is needed to understand the motivational factors behind healthy dietary practices during the pandemic and whether pre-pandemic awareness and practices likely led to a positive influence.

It is well known that any amount of physical activity, no matter how little, is beneficial. Sedentary behavior associated with excessive television watching, increased internet browsing time, playing screen games, and using mobile devices are shown to negatively impact chronic disease outcomes ${ }^{77}$. With parks and gymnasiums closed and public movement in the open discouraged, the ability of people to remain physically active has proven to be challenging during the lockdown period. Though the majority of studies have shown a reduction in physical activity levels and increased sedentariness during the lockdown, it is heartening to note that some individuals in select settings have seen an opportunity to be creative in finding ways of staying even minimally active (e.g., gardening, exploring online free exercise routines, and walking up and down the stairs/steps) in a home environment ${ }^{19,78}$.

Adequate sleep improves concentration and diligent decision-making. Disrupted and suboptimal sleep on the other hand has been correlated with an elevated body mass index, obesity, metabolic syndrome, and type 2 diabetes ${ }^{79}$. Exposure to blue light also increases nighttime heart rate, blood pressure, and core body temperature, and suppresses melatonin and sleepiness. The high prevalence of insomnia and an increase in screen time during the lockdown periods are concerning and a reversal of some of these newfound habits will be 
important in order to promote sleep hygiene in communities and reap the benefits of sleep in improving mood, immunity, and overall health and well-being of individuals and communities. Reports of people hoarding cigarettes and alcohol along with food and toilet paper during the early phases of the pandemic are suggestive of the fact that smoking cessation and quitting alcohol may not have been a priority during the lockdown period; our review confirms this. Some smokers wanting to quit may have found accessing smoking cessation clinics a challenge due to suspension of non-essential health services, as have those dependent on alcohol and other psychoactive substances. Getting substance cessation and control programs back on track at local, national, and international levels will require a doubling of efforts as countries start relaxing lockdown measures.

Most of the studies in our review have unequivocally pointed towards high stress levels among populations worldwide. Stress is causally associated with risky behaviors, such as smoking, physical inactivity, and heavy alcohol use, all of which are independent risk factors for chronic diseases - leading to additional concern. However, on the positive side, staying at home provides an opportunity for families to bond and engage in activities at their convenience, which can elicit relaxation and calming responses. Examples of such activities include prayers, meditation, and breathing exercises, all having positive health benefits ${ }^{80}$. Nevertheless, we did not find any studies where communities claimed to be using such positive coping measures to alleviate stress. This may indicate a lack of awareness in communities regarding the importance of healthy lifestyle measures for stress management, as well as, public health professionals/healthcare practitioners not emphasizing the importance of addressing lifestylerelated factors adequately.

More importantly, one cannot deny that of all the altered lifestyle factors, the crucial one has been the loss of connectedness and social networking. This is unfortunate since positive resonance and micro-moments of connectivity encountered in situations of togetherness are associated with health and longevity. Virtual connectivity, though inadequately studied, and small acts of kindness where possible during this period, remain the best measures of improving relationships and connectedness. The lockdown has no doubt led to an increase in family time, at least for nuclear families in most instances. This may have been an opportune time for families to bond and strengthen relationships. One concern of staying home, however, has been reports of increased domestic violence. Future research is likely to provide more answers to the questions surrounding the nature of relationships and domestic activities, and their overall impact--positive or negative--on mental and physical health.

During this period, patients with pre-existing NCDs have suffered both from their inability to maintain a healthy lifestyle and neglect from national health authorities and hospitals with guidance issued to delay routine care and non-urgent procedures. Since an advisory by the US Medicare and Medicaid Centers in mid-March to delay non-urgent procedures, adult patient visits for primary care and gastroenterology services declined by $49 \%$ and $61 \%$ respectively ${ }^{81}$. This could, inevitably, compromise patients receiving timely care. Tapper and Asrani ${ }^{82}$ discuss care in the context of managing patients with liver cirrhosis (a common complication of obesity and diabetes) and suggest three challenging phases in treating patients with chronic disease: 1) the current 'intense period' with a delay in routine care 2) a 'return to the norm' when physical distancing ends and health systems respond to a backlog of cases, and 3) a 'protracted period' of poor outcomes due to unabated disease progression. The world is headed towards a catch-up period with respect to NCDs due to altered lifestyles and concomitant treatment backlog.

The American College of Lifestyle Medicine proposes healthy lifestyle measures to reduce chronic disease risk and improve immunity during this period ${ }^{1}$ (Table 2). Though the measures may appear relatively easy and simple enough to implement, many people may not adhere to these recommendations for a variety of reasons, including poverty, lack of education, 
unaffordability of technological resources, cramped living spaces, and poor city planning. Governments, health care practitioners and communities should do everything possible to break those barriers and ensure universal access to health lifestyle measures for all.

Table 2. Healthy lifestyle measures to reduce chronic disease risk and improve immunity [1]

- Stress management by practicing techniques like meditation and yoga.

- Healthy eating focused on a plant-based diet and whole grains.

- Remaining physically active.

- Quitting smoking and harmful substances.

- Optimizing good sleep hygiene.

- Maintaining social connections virtually like video or phone calls with friends and families.

\section{Strengths and Limitations}

This narrative review is a comprehensive analysis of the existing literature on the impact of COVID-19 related lockdown on the lifestyles of people globally. As the review is narrative, we included all the relevant and available published literature on the subject to ensure comprehensiveness. We have not done a quality assessment of each of the publications and instead used data as reported in the articles. Most of the studies included in our review are observational, which is an inherent limitation of the review study design. Despite this general limitation--as is the case with narrative reviews--we do not consider this a major impediment to our research pursuit, as our analysis relies largely on trends and qualitative aspects of data. We also acknowledge that home quarantine, institutional quarantine, community quarantine, and isolation can have different levels of impact on lifestyles, but we did not find adequate number of studies in each category to analyze them separate

\section{Conclusion}

COVID-19 has disrupted our lifestyle balance. Based on its rapid assessment of service delivery for NCDs in 163 countries, the World Health Organization has identified that the COVID-19 pandemic could throw the global health progress on NCDs off track ${ }^{83}$. This requires that we act as one world and make every effort to promote healthy lifestyles in communities worldwide. COVID-19 will likely redefine conventional public health by placing additional emphasis on healthy lifestyles, disease prevention, and self-care. Given the strong evidence for lifestyle medicine, incorporating lifestyle medicine in day-to-day medical practice is likely to receive worldwide attention. Serious consideration should be given to augment professional health curricula by including topics such as evidence-based public health practices, lifestyle medicine, and building health systems that can address the holistic needs of local populations before, during, and after crisis situations. Also, integrating basic health, infectious disease and lifestyle education in school and undergraduate college education would be a step in the right direction. It would be up to the governments, communities, and healthcare delivery systems to learn and benefit from the lifestyle medicine lessons during and consequent to the COVID-19 pandemic--with the ultimate objective of promoting healthy lifestyles in communities. 


\section{ACKNOWLEDGMENTS}

We would like to thank Silva Kouyoumjian, Clinical Research Coordinator, Tobacco Control Center, Hamad Medical Corporation, Qatar for a friendly peer review of the manuscript, Cathleen de Groot, Manager, User Service, Distributed eLibrary Weill Cornell MedicineQatar for her editing and formatting services and Anupama Jithesh, Projects Coordinator, Institute for Population Health, Weill Cornell Medicine-Qatar for assisting in data tabulation.

\section{AUTHOR CONTRIBUTIONS}

Sathyanarayanan Doraiswamy (SD), Sohaila Cheema (SC), Ahmad Al Mulla (AM), and Ravinder Mamtani (RM) collectively contributed to the conception of the study. SD carried out the literature search, screening, and data extraction. Analysis and manuscript drafting were carried out by SD with support from SC, AM and RM. All authors read, edited, and approved the final manuscript.

\section{DISCLOSURE STATEMENT}

The authors report no potential conflicts of interest.

\section{ETHICS AND CONSENT}

Not applicable

\section{FUNDING INFORMATION}

No funding was received for this work

\section{Annex 1:}

$\underline{\text { SANRA - a scale for the quality assessment of narrative review articles }}$

1) Justification of the article's importance of the readership - Page 4

2) Statement of concrete aims or formulation of questions - Page 4

3) Description of the literature search - Page 6

4) Referencing - Pages 6 - 16

5) Scientific reasoning - Pages 17-22

6) Appropriate presentation of data - Pages 6-16 


\section{References}

1. Mauriello LA, Kristi. Lifestyle Medicine is essential, now more than ever, (2020, accessed 6 April 2020).

2. Green BN, Johnson CD and Adams A. Writing narrative literature reviews for peerreviewed journals: secrets of the trade. Journal of chiropractic medicine 2006; 5: 101-117. DOI: 10.1016/S0899-3467(07)60142-6.

3. Baethge C, Goldbeck-Wood S and Mertens S. SANRA-a scale for the quality assessment of narrative review articles. Research Integrity and Peer Review 2019; 4: 5. DOI: 10.1186/s41073-019-0064-8.

4. Hupe M. EndNote X9. Journal of Electronic Resources in Medical Libraries 2019; 16: 117-119.

5. Górnicka M, Drywień ME, Zielinska MA, et al. Dietary and Lifestyle Changes During COVID-19 and the Subsequent Lockdowns among Polish Adults: A Cross-Sectional Online Survey PLifeCOVID-19 Study. Nutrients 2020; 12 2020/08/07. DOI: 10.3390/nu12082324.

6. Sidor A and Rzymski P. Dietary Choices and Habits during COVID-19 Lockdown: Experience from Poland. Nutrients 2020; 12 2020/06/07. DOI: 10.3390/nu12061657.

7. Scarmozzino F and Visioli F. Covid-19 and the Subsequent Lockdown Modified Dietary Habits of Almost Half the Population in an Italian Sample. Foods 2020; 9 2020/05/30. DOI: $10.3390 /$ foods 9050675 .

8. Shammi M, Bodrud-Doza M, Towfiqul Islam ARM, et al. COVID-19 pandemic, socioeconomic crisis and human stress in resource-limited settings: A case from Bangladesh. Heliyon 2020; 6: e04063. 2020/05/29. DOI: 10.1016/j.heliyon.2020.e04063.

9. Ammar A, Brach M, Trabelsi K, et al. Effects of COVID-19 Home Confinement on Eating Behaviour and Physical Activity: Results of the ECLB-COVID19 International Online Survey. Nutrients 2020; 12 2020/06/03. DOI: 10.3390/nu12061583.

10. Pietrobelli A, Pecoraro L, Ferruzzi A, et al. Effects of COVID-19 Lockdown on Lifestyle Behaviors in Children with Obesity Living in Verona, Italy: A Longitudinal Study. Obesity (Silver Spring) 2020 2020/05/01. DOI: 10.1002/oby.22861.

11. Pellegrini M, Ponzo V, Rosato R, et al. Changes in Weight and Nutritional Habits in Adults with Obesity during the "Lockdown" Period Caused by the COVID-19 Virus Emergency. Nutrients 2020; 12 2020/07/11. DOI: 10.3390/nu12072016.

12. Zhao A, Li Z, Ke Y, et al. Dietary Diversity among Chinese Residents during the COVID-19 Outbreak and Its Associated Factors. Nutrients 2020; 12 2020/06/11. DOI: 10.3390/nu12061699.

13. Rodríguez-Pérez C, Molina-Montes E, Verardo V, et al. Changes in Dietary Behaviours during the COVID-19 Outbreak Confinement in the Spanish COVIDiet Study. Nutrients 2020; 12 2020/06/14. DOI: 10.3390/nu12061730.

14. Ruiz-Roso MB, de Carvalho Padilha P, Mantilla-Escalante DC, et al. Covid-19 Confinement and Changes of Adolescent's Dietary Trends in Italy, Spain, Chile, Colombia and Brazil. Nutrients 2020; 12 2020/06/21. DOI: 10.3390/nu12061807.

15. Cooper M, Reilly EE, Siegel JA, et al. Eating disorders during the COVID-19 pandemic and quarantine: an overview of risks and recommendations for treatment and early intervention. Eat Disord 2020: 1-23. 2020/07/10. DOI: 10.1080/10640266.2020.1790271.

16. He M, Xian Y, Lv X, et al. Changes in Body Weight, Physical Activity, and Lifestyle During the Semi-lockdown Period After the Outbreak of COVID-19 in China: An Online Survey. Disaster Med Public Health Prep 2020: 1-6. 2020/07/15. DOI: 10.1017/dmp.2020.237.

17. Cancello R, Soranna D, Zambra G, et al. Determinants of the Lifestyle Changes during COVID-19 Pandemic in the Residents of Northern Italy. Int J Environ Res Public Health 2020; 17 2020/09/03. DOI: 10.3390/ijerph17176287. 
18. Meyer J, McDowell C, Lansing J, et al. Changes in Physical Activity and Sedentary Behavior in Response to COVID-19 and Their Associations with Mental Health in 3052 US Adults. Int J Environ Res Public Health 2020; 17 2020/09/10. DOI: 10.3390/ijerph17186469. 19. Constandt B, Thibaut E, De Bosscher V, et al. Exercising in Times of Lockdown: An Analysis of the Impact of COVID-19 on Levels and Patterns of Exercise among Adults in Belgium. International journal of environmental research and public health 2020; 17 2020/06/14. DOI: 10.3390/ijerph17114144.

20. Ong JL, Lau T, Massar SAA, et al. COVID-19 Related Mobility Reduction: Heterogenous Effects on Sleep and Physical Activity Rhythms. Sleep 2020 2020/09/13. DOI: $10.1093 /$ sleep/zsaa 179 .

21. Zhang SX, Wang Y, Rauch A, et al. Unprecedented disruptions of lives and work: Health, distress and life satisfaction of working adults in China one month into the COVID-19 outbreak. 2020. DOI: 10.1101/2020.03.13.20034496.

22. Park S, Kim B and Lee J. Social Distancing and Outdoor Physical Activity During the COVID-19 Outbreak in South Korea: Implications for Physical Distancing Strategies. Asia Pac J Public Health 2020: 1010539520940929. 2020/07/16. DOI: 10.1177/1010539520940929.

23. Aung MN, Yuasa M, Koyanagi Y, et al. Sustainable health promotion for the seniors during COVID-19 outbreak: a lesson from Tokyo. Journal of infection in developing countries 2020; 14: 328-331. 2020/05/08. DOI: 10.3855/jidc.12684.

24. Schuch FB, Bulzing RA, Meyer J, et al. Associations of moderate to vigorous physical activity and sedentary behavior with depressive and anxiety symptoms in self-isolating people during the COVID-19 pandemic: A cross-sectional survey in Brazil. Psychiatry Res 2020; 292: 113339. 2020/08/04. DOI: 10.1016/j.psychres.2020.113339.

25. Beck F, Léger D, Fressard L, et al. Covid-19 health crisis and lockdown associated with high level of sleep complaints and hypnotic uptake at the population level. J Sleep Res 2020: e13119. 2020/07/01. DOI: 10.1111/jsr.13119.

26. Marelli S, Castelnuovo A, Somma A, et al. Impact of COVID-19 lockdown on sleep quality in university students and administration staff. $J$ Neurol 2020: 1-8. 2020/07/13. DOI: 10.1007/s00415-020-10056-6.

27. Marelli S, Castelnuovo A, Somma A, et al. Impact of COVID-19 lockdown on sleep quality in university students and administration staff. $J$ Neurol 2020 2020/07/13. DOI: 10.1007/s00415-020-10056-6.

28. Majumdar P, Biswas A and Sahu S. COVID-19 pandemic and lockdown: cause of sleep disruption, depression, somatic pain, and increased screen exposure of office workers and students of India. Chronobiol Int 2020: 1-10. 2020/07/15. DOI: 10.1080/07420528.2020.1786107.

29. Cellini N, Canale N, Mioni G, et al. Changes in sleep pattern, sense of time and digital media use during COVID-19 lockdown in Italy. J Sleep Res 2020: e13074. 2020/05/16. DOI: $10.1111 /$ jsr.13074.

30. Sinha M, Pande B and Sinha R. Impact of COVID-19 lockdown on sleep-wake schedule and associated lifestyle related behavior: A national survey. J Public Health Res 2020; 9: 1826. 2020/09/03. DOI: 10.4081/jphr.2020.1826.

31. Cajochen C, Munch M, Kobialka S, et al. High sensitivity of human melatonin, alertness, thermoregulation, and heart rate to short wavelength light. J Clin Endocrinol Metab 2005; 90: 1311-1316. 2004/12/09. DOI: 10.1210/jc.2004-0957.

32. Altena E, Baglioni C, Espie CA, et al. Dealing with sleep problems during home confinement due to the COVID-19 outbreak: Practical recommendations from a task force of the European CBT-I Academy. J Sleep Res 2020: e13052. 2020/04/05. DOI: $10.1111 /$ jsr.13052. 
33. Blume C, Schmidt MH and Cajochen C. Effects of the COVID-19 lockdown on human sleep and rest-activity rhythms. Current Biology 2020.

34. Wright KP, Linton SK, Withrow D, et al. Sleep in University Students Prior to and During COVID-19 Stay-at-Home Orders. Current Biology 2020.

35. Gao C and Scullin MK. Sleep health early in the coronavirus disease 2019 (COVID19) outbreak in the United States: integrating longitudinal, cross-sectional, and retrospective recall data. Sleep Med 2020; 73: 1-10. 2020/08/04. DOI: 10.1016/j.sleep.2020.06.032.

36. Klemperer EM, West JC, Peasley-Miklus C, et al. Change in Tobacco and Electronic Cigarette Use and Motivation to Quit in Response to COVID-19. Nicotine \& tobacco research : official journal of the Society for Research on Nicotine and Tobacco 2020; 22: 1662-1663. DOI: $10.1093 / \mathrm{ntr} / \mathrm{ntaa} 072$.

37. Yach D. Tobacco Use Patterns in Five Countries During the COVID-19 Lockdown. Nicotine Tob Res 2020; 22: 1671-1672. 2020/05/28. DOI: 10.1093/ntr/ntaa097.

38. Gretler C. Stay-at-Home Smoking Helps Tobacco Sales During Virus Lockdowns. Bloomberg, 2020.

39. Klemperer EM, West JC, Peasley-Miklus C, et al. Change in tobacco and electronic cigarette use and motivation to quit in response to COVID-19. Nicotine \& tobacco research : official journal of the Society for Research on Nicotine and Tobacco 2020: ntaa072. DOI: $10.1093 / \mathrm{ntr} / \mathrm{ntaa} 072$.

40. Caponnetto P, Inguscio L, Saitta C, et al. Smoking behavior and psychological dynamics during COVID-19 social distancing and stay-at-home policies: A survey. Health psychology research 2020; 8: 9124-9124. DOI: 10.4081/hpr.2020.9124.

41. Chodkiewicz J, Talarowska M, Miniszewska J, et al. Alcohol Consumption Reported during the COVID-19 Pandemic: The Initial Stage. Int J Environ Res Public Health 2020; 17 2020/07/03. DOI: 10.3390/ijerph17134677.

42. Balhara YPS, Singh S and Narang P. Effect of lockdown following COVID-19 pandemic on alcohol use and help-seeking behavior: Observations and insights from a sample of alcohol use disorder patients under treatment from a tertiary care center. Psychiatry and clinical neurosciences 2020: 10.1111/pcn.13075. DOI: 10.1111/pcn.13075.

43. Hobin E and Smith B. Is another public health crisis brewing beneath the COVID-19 pandemic? Canadian journal of public health = Revue canadienne de sante publique 2020; 111: 392-396. 2020/06/18. DOI: 10.17269/s41997-020-00360-z.

44. Sun Y, Li Y, Bao Y, et al. Brief Report: Increased Addictive Internet and Substance Use Behavior During the COVID-19 Pandemic in China. Am J Addict 2020; 29: 268-270. 2020/06/06. DOI: 10.1111/ajad.13066.

45. The Lancet Gastroenterology H. Drinking alone: COVID-19, lockdown, and alcoholrelated harm. Lancet Gastroenterol Hepatol 2020; 5: 625. 2020/06/20. DOI: 10.1016/s24681253(20)30159-x.

46. Neill E, Meyer D, Toh WL, et al. Alcohol use in Australia during the early days of the COVID-19 pandemic: Initial results from the COLLATE project. Psychiatry Clin Neurosci 2020 2020/07/01. DOI: 10.1111/pcn.13099.

47. Addiction EMCfDaD. COVID-19 and people who use drugs, http://www.emcdda.europa.eu/publications/topic-overviews/covid-19-and-people-who-usedrugs en (2020, accessed 06 April 2020).

48. Dietze PM and Peacock A. Illicit drug use and harms in Australia in the context of COVID-19 and associated restrictions: Anticipated consequences and initial responses. Drug and alcohol review 2020; 39: 297-300. DOI: 10.1111/dar.13079.

49. Rolland B, Haesebaert F, Zante E, et al. Global changes and factors of increase in caloric/salty food, screen, and substance use, during the early COVID-19 containment phase 
in France: a general population online survey. JMIR Public Health Surveill 2020 2020/06/27. DOI: $10.2196 / 19630$.

50. Lima CKT, Carvalho PMM, Lima I, et al. The emotional impact of Coronavirus 2019nCoV (new Coronavirus disease). Psychiatry Res 2020; 287: 112915. 2020/03/22. DOI: 10.1016/j.psychres.2020.112915.

51. Cao W, Fang Z, Hou G, et al. The psychological impact of the COVID-19 epidemic on college students in China. Psychiatry Res 2020; 287: 112934. 2020/03/20. DOI: 10.1016/j.psychres.2020.112934.

52. Holt-Lunstad J. The Potential Public Health Relevance of Social Isolation and Loneliness: Prevalence, Epidemiology, and Risk Factors. Public Policy \& Aging Report 2018; 27: 127-130. DOI: 10.1093/ppar/prx030.

53. Herat M. "I feel like death on legs": COVID-19 isolation and mental health. Social Sciences \& Humanities Open 2020; 2: $100042 . \quad$ DOI: https://doi.org/10.1016/j.ssaho.2020.100042.

54. Rossi R, Socci V, Talevi D, et al. COVID-19 Pandemic and Lockdown Measures Impact on Mental Health Among the General Population in Italy. Front Psychiatry 2020; 11: 790. 2020/08/28. DOI: 10.3389/fpsyt.2020.00790.

55. Lu H, Nie P and Qian L. Do Quarantine Experiences and Attitudes Towards COVID19 Affect the Distribution of Mental Health in China? A Quantile Regression Analysis. Appl Res Qual Life 2020: 1-18. 2020/08/25. DOI: 10.1007/s11482-020-09851-0.

56. Croizier C, Bouillon-Minois JB, Bay JO, et al. COVID-19 lockdown and mental health: why we must look into oncology units. Psychol Med 2020: 1-2. 2020/07/04. DOI: $10.1017 / \mathrm{s} 0033291720002500$.

57. Lebel C, MacKinnon A, Bagshawe M, et al. Elevated depression and anxiety symptoms among pregnant individuals during the COVID-19 pandemic. J Affect Disord 2020; 277: 5-13. 2020/08/11. DOI: 10.1016/j.jad.2020.07.126.

58. Rettie H and Daniels J. Coping and tolerance of uncertainty: Predictors and mediators of mental health during the COVID-19 pandemic. Am Psychol 2020 2020/08/04. DOI: 10.1037/amp0000710.

59. Caballero-Domínguez CC, Jiménez-Villamizar MP and Campo-Arias A. Suicide risk during the lockdown due to coronavirus disease (COVID-19) in Colombia. Death Stud 2020: 1-6. 2020/06/27. DOI: 10.1080/07481187.2020.1784312.

60. Dsouza DD, Quadros S, Hyderabadwala ZJ, et al. Aggregated COVID-19 suicide incidences in India: Fear of COVID-19 infection is the prominent causative factor. Psychiatry Res 2020; 290: 113145. 2020/06/17. DOI: 10.1016/j.psychres.2020.113145.

61. Fullana MA, Hidalgo-Mazzei D, Vieta E, et al. Coping behaviors associated with decreased anxiety and depressive symptoms during the COVID-19 pandemic and lockdown. $J$ Affect Disord 2020; 275: 80-81. 2020/07/14. DOI: 10.1016/j.jad.2020.06.027.

62. Al-Qahtani AM, Elgzar WT and Ibrahim HA. COVID-19 Pandemic: Psycho-social Consequences During the Social Distancing Period Among Najran City Population. Psychiatr Danub 2020; 32: 280-286. 2020/08/17. DOI: 10.24869/psyd.2020.280.

63. Ammar A, Chtourou H, Boukhris O, et al. COVID-19 Home Confinement Negatively Impacts Social Participation and Life Satisfaction: A Worldwide Multicenter Study. Int J Environ Res Public Health 2020; 17 2020/09/02. DOI: 10.3390/ijerph17176237.

64. Ogden RS. The passage of time during the UK Covid-19 lockdown. PLoS One 2020; 15: e0235871. 2020/07/07. DOI: 10.1371/journal.pone.0235871.

65. Imran N, Aamer I, Sharif MI, et al. Psychological burden of quarantine in children and adolescents: A rapid systematic review and proposed solutions. Pak J Med Sci 2020; 36: 11061116. 2020/07/25. DOI: 10.12669/pjms.36.5.3088. 
66. Kroshus E. Gender, marital status, and commercially prepared food expenditure. J Nutr Educ Behav 2008; 40: 355-360. 2008/11/06. DOI: 10.1016/j.jneb.2008.05.012.

67. Evans ML, Lindauer M and Farrell ME. A Pandemic within a Pandemic - Intimate Partner Violence during Covid-19. New England Journal of Medicine 2020. DOI: 10.1056/NEJMp2024046.

68. Saqib MAN, Siddiqui S, Qasim M, et al. Effect of COVID-19 lockdown on patients with chronic diseases. Diabetes Metab Syndr 2020; 14: 1621-1623. 2020/09/06. DOI: 10.1016/j.dsx.2020.08.028.

69. Ghosh A, Arora B, Gupta R, et al. Effects of nationwide lockdown during COVID-19 epidemic on lifestyle and other medical issues of patients with type 2 diabetes in north India. Diabetes Metab Syndr 2020; 14: 917-920. 2020/06/24. DOI: 10.1016/j.dsx.2020.05.044.

70. Ruiz-Roso MB, Knott-Torcal C, Matilla-Escalante DC, et al. COVID-19 Lockdown and Changes of the Dietary Pattern and Physical Activity Habits in a Cohort of Patients with Type 2 Diabetes Mellitus. Nutrients 2020; 12 2020/08/08. DOI: 10.3390/nu12082327.

71. Cransac-Miet A, Zeller M, Chagué F, et al. Impact of COVID-19 lockdown on lifestyle adherence in stay-at-home patients with chronic coronary syndromes: Towards a time bomb. Int J Cardiol 2020 2020/09/06. DOI: 10.1016/j.ijcard.2020.08.094.

72. Kang C, Yang S, Yuan J, et al. Patients with chronic illness urgently need integrated physical and psychological care during the COVID-19 outbreak. Asian journal of psychiatry 2020; 51: 102081-102081. DOI: 10.1016/j.ajp.2020.102081.

73. Nutrition UNSSCo. The COVID-19 pandemic is disrupting people's food environments: a resource list on Food Systems and Nutrition responses - UNSCN, https://www.unscn.org/en/news-events/recent-news?idnews $=2039$ (2020, accessed 06 April 2020).

74. FAO I, UNICEF, WFP and WHO. The State of Food Security and Nutrition in the World 2020. 2020. Rome.

75. Di Renzo L, Gualtieri P, Pivari F, et al. Eating habits and lifestyle changes during COVID-19 lockdown: an Italian survey. J Transl Med 2020; 18: 229. 2020/06/10. DOI: 10.1186/s12967-020-02399-5.

76. Association AH. How to Cook Healthier at Home, https://www.heart.org/en/healthyliving/healthy-eating/cooking-skills/cooking/how-to-cook-healthier-at-home (2020, accessed 06 April 2020).

77. Matthews CE, George SM, Moore SC, et al. Amount of time spent in sedentary behaviors and cause-specific mortality in US adults. Am J Clin Nutr 2012; 95: 437-445. 2012/01/06. DOI: 10.3945/ajen.111.019620.

78. Ding D, Del Pozo Cruz B, Green MA, et al. Is the COVID-19 lockdown nudging people to be more active: a big data analysis. Br J Sports Med 2020 2020/07/02. DOI: 10.1136/bjsports-2020-102575.

79. Ferrie JE, Kivimaki M, Akbaraly TN, et al. Change in Sleep Duration and Type 2 Diabetes: The Whitehall II Study. Diabetes Care 2015; 38: 1467-1472. 2015/06/13. DOI: 10.2337/dc15-0186.

80. Harmon RL and Myers MA. Prayer and meditation as medical therapies. Phys Med Rehabil Clin N Am 1999; 10: 651-662. 1999/10/12.

81. Issaka RB and Somsouk M. Colorectal Cancer Screening and Prevention in the COVID-19 Era. In: JAMA Health Forum 2020, pp.e200588-e200588. American Medical Association.

82. Tapper EB and Asrani SK. COVID-19 pandemic will have a long-lasting impact on the quality of cirrhosis care. J Hepatol 2020 2020/04/17. DOI: 10.1016/j.jhep.2020.04.005.

83. World Health O. Rapid assessment of service delivery for NCDs during COVID-19 pandemic. Geneva: World Health Organization 2020. 
